\title{
Excitation of axisymmetric and non-axisymmetric guided waves in elastic hollow cylinders by magnetostrictive transducers
}

\author{
Xiao-wei ZHANG ${ }^{1}$, Zhi-feng TANG ${ }^{\dagger ‡ 2}$, Fu-zai LV ${ }^{1}$, Xiao-hong PAN ${ }^{1}$ \\ ( ${ }^{1}$ Institute of Modern Manufacture Engineering, Zhejiang University, Hangzhou 310027, China) \\ ('Institute of Advanced Digital Technologies and Instrumentation, Zhejiang University, Hangzhou 310027, China) \\ †E-mail: tangzhifeng@zju.edu.cn \\ Received June 21, 2015; Revision accepted Oct. 13, 2015; Crosschecked Feb. 15, 2016
}

\begin{abstract}
Ultrasonic guided waves have been successfully applied in nondestructive evaluation (NDE) and structural health monitoring (SHM) of pressure vessels and pipelines due to their advantages, such as long detection range and high inspection efficiency. Compared with other ultrasonic guided wave actuators, magnetostrictive transducers are more cost-effective, involve simpler fabrication process, and have higher possible transduction efficiency. The normal mode expansion (NME) method is adopted to analyze the forced response and perturbation analysis of elastic hollow cylinders with respect to magnetostrictive loadings, including partial loading, axial array loading, and circular array loading. The phase velocity and frequency spectra of axisymmetric/non-axisymmetric guided waves excited by magnetostrictive transducers are analyzed. The theoretically predicted trends are verified by finite element numerical simulations and experiments.
\end{abstract}

Key words: Guided waves, Hollow cylinder, Normal mode expansion (NME), Magnetostriction http://dx.doi.org/10.1631/jzus.A1500184 CLC number: TB559; O347

\section{Introduction}

Ultrasonic guided waves have already been demonstrated by various research works to have great potential and a good prospect in online nondestructive evaluation (NDE) and long-term structural health monitoring (SHM) of pipelines of various industries (Rose, 2014) due to its various corresponding advantages, such as single-point excitation, long detection range, high inspection efficiency, and $100 \%$ cross-sectional detectability. The three main types of transducers that are currently in service for guided wave inspection of pipelines are piezoelectric

\footnotetext{
${ }^{\ddagger}$ Corresponding author

* Project supported by the National Natural Science Foundation of China (Nos. 61271084 and 51275454), and the Fundamental Research Funds for the Central Universities, China

(ib) ORCID: Xiao-wei ZHANG, http://orcid.org/0000-0002-3995-5950; Zhi-feng TANG, http://orcid.org/0000-0001-5591-8175

(c) Zhejiang University and Springer-Verlag Berlin Heidelberg 2016
}

transducers (Alleyne and Cawley, 1996; Marty, 2002), magnetostrictive transducers (Kwun and Bartels, 1998; Kim Y.Y. et al., 2005; Cho et al., 2006; Turcu, 2008; Lee et al., 2009; Kim Y.G. et al., 2011), and electromagnetic acoustic transducers (Ribichini, 2011). Magnetostrictive transducers are more cost-effective, involve relatively simpler fabrication process, and have higher transduction efficiency than other types of transducers. However, it is highly important to develop a theoretical approach to consider the forced response and perturbation analysis of elastic pipes under magnetostrictive loadings, and the excitation of axisymmetric and non-axisymmetric guided waves in elastic hollow cylinders by finite size magnetostrictive transducers, with the unique feature of magnetostriction taken into consideration. These topics are rarely reported in the literature, but further investigation can provide guidance for the further development of magnetostrictive guided wave transducers.

Problems involving the forced response of a structure are typically solved using one of the 
following two approaches (Rose, 2014): integral transform technique or normal mode expansion (NME) method. The integral transform technique solves the guided wave excitations through transforms, such as Laplace, Fourier, Hankel, and Mellin. The NME method, on the other hand, analyzes the wave fields in the cylinder in the form of a sum of an infinite number of normal modes. Gazis (1959) showed that there exist an infinite number of normal modes in an elastic hollow cylinder, each with its own characteristics, such as phase velocity, group velocity, and wave structure profile. He obtained the general solution of harmonic waves propagating in an infinite long hollow cylinder, which has been very beneficial for long-range guided wave inspection on widely distributed pipelines. The forced response problem in a hollow cylinder problem was first studied by Ditri and Rose (1992) using the NME method to obtain the amplitude factors of different guided wave modes. Li and Rose (2001a) investigated the field distribution of non-axisymmetric longitudinal waves. The angular profile was calculated by taking into account the amplitude factors of every excited mode. The NME method was also used by Luo (2005), Zhang (2005), Mu (2008), and $\mathrm{Mu}$ and Rose (2008) in their studies. Several results have been reported on the source influence analysis of hollow cylinders with respect to piezoelectric loadings. However, a comprehensive perturbation analysis on pipes with respect to magnetostrictive loadings, considering unique features of magnetostriction, is essential for further development of guided wave magnetostrictive transducers and transducer arrays.

In this studies, the classical NME method is adopted to study the excitation of axisymmetric and non-axisymmetric guided wave modes in linear elastic hollow cylinders by magnetostrictive actuation. The phase velocity and frequency spectra of axisymmetric and non-axisymmetric guided wave modes excited by magnetostrictive transducers are modeled with a large static bias magnetic field assumption. The influence of load parameters is analyzed. Several novel results have been found about the source influence of different magnetostrictive loadings. The prediction of guided wave excitation by the NME method is verified by finite element numerical simulations and experiments.

\section{NME method}

\subsection{Normal modes of a hollow cylinder}

The NME method solving the forced loading problem is analogous to the eigenfunction expansion method in the field of mathematics, and the normal modes of cylinders serve as the eigenfunctions. The main idea of the NME method is to assume that the sought function can be written in the form of a series of known functions, i.e., the normal modes, each with an unknown amplitude. The goal is then to find a general expression for the unknown amplitudes or a numerical estimate of them. Note that the efficiency of NME method depends on two main considerations: completeness and orthogonality of the normal modes (Rose, 2014).

Consider a hollow cylinder in a cylindrical coordinate system as shown in Fig. 1. There exists an infinite number of propagating modes in the hollow cylinder. The velocity field due to a normal mode with circumferential order $N$ in the $m$ th family can be written as (Ditri and Rose, 1992; Li and Rose, 2001a):

$$
\begin{aligned}
\boldsymbol{v}_{m}^{N}(r, \theta) \mathrm{e}^{\mathrm{i}\left(\omega t-k_{m}^{N} z\right)}= & \left\{R_{m r}^{N}(r) \phi_{r}^{N}(N \theta) \boldsymbol{e}_{r}\right. \\
& +R_{m \theta}^{N}(r) \phi_{\theta}^{N}(N \theta) \boldsymbol{e}_{\theta} \\
& \left.+R_{m z}^{N}(r) \phi_{z}^{N}(N \theta) \boldsymbol{e}_{z}\right\} \mathrm{e}^{\mathrm{i}\left(\omega t-k_{m}^{N} z\right)},
\end{aligned}
$$

where $\omega$ and $k$ are the angular frequency and wave number, respectively. Functions $R(r)$ and $\phi(N \theta)$ denote the radical and angular field distributions of the velocity component of the normal mode with circumferential order $N$ in the $m$ th family, respectively. The phase velocity dispersion curves of an elastic pipe with the parameters given in Table 1 solved using the semi-analytical finite element method (Marzani, 2008; Zhang et al., 2014) for the axisymmetric and non-axisymmetric modes are shown in Fig. 2. Note that the curves in different colors denote modes with different circumferential orders.

According to the NME method, the generated particle velocity can then be expanded as

$$
\boldsymbol{v} \mathrm{e}^{\mathrm{i} \omega t}=\sum_{N, \mathrm{~m}} A_{m}^{N} \boldsymbol{v}_{m}^{N} \mathrm{e}^{\mathrm{i} \omega t}
$$


where $A_{m}^{N}$ is the NME amplitude of the mode with circumferential order $N$ in the $m$ th family containing wave component $\mathrm{e}^{-\mathrm{i} k_{m}^{N} z}$.

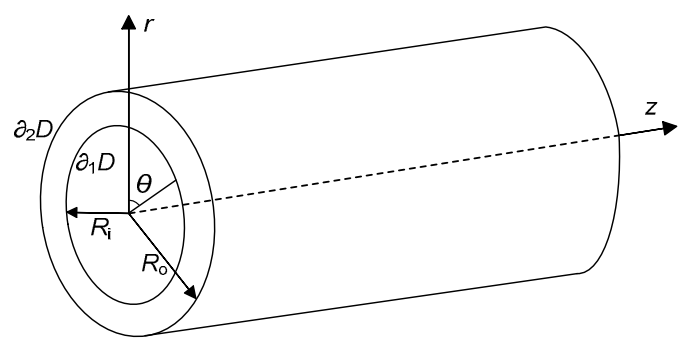

Fig. 1 Stress-free hollow cylinder in a cylindrical coordinate system

$R_{\mathrm{i}}$ and $R_{\mathrm{o}}$ are the inner and outer radii, and $\partial_{1} D$ and $\partial_{2} D$ are the inner and outer boundaries, respectively

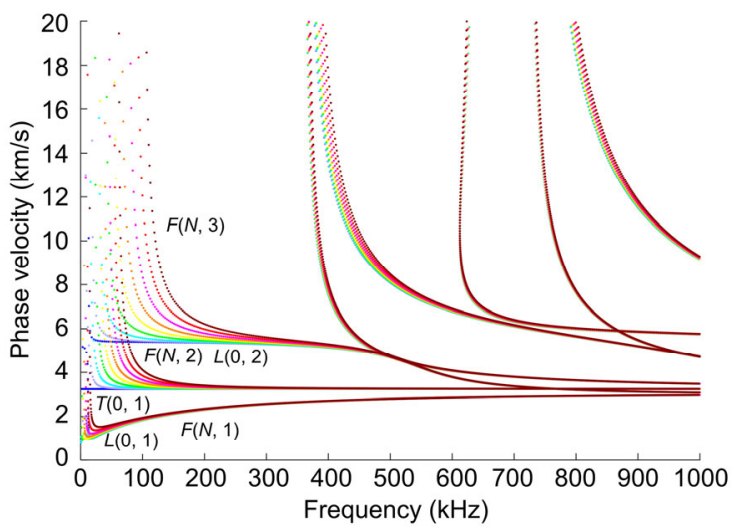

Fig. 2 Phase velocity dispersion curves for $L(0, m), T(0$, $m)$, and $F(N, m)$ modes in a steel pipe. Note: for interpretation of the references to color in this figure, the reader is referred to the web version of this article

\subsection{Orthogonality of normal modes}

Before calculating the amplitude factor $A_{m}^{N}$ by using the NME method, the orthogonality relation between the normal modes should be established. We start with the complex reciprocity relation (Auld, 1990), which is described as

$$
\nabla \cdot\left(\boldsymbol{v}_{2}^{*} \cdot \boldsymbol{T}_{1}+\boldsymbol{v}_{1} \cdot \boldsymbol{T}_{2}^{*}\right)=0
$$

where $\boldsymbol{v}$ and $\boldsymbol{T}$ represent the particle velocity and stress field of a solution to the linear elastic wave propagation equation, respectively. Let $\boldsymbol{v}_{1}$ and $\boldsymbol{T}_{1}, \boldsymbol{v}_{2}$ and $\boldsymbol{T}_{2}$ denote different normal modes of the cylinder:

$$
\begin{cases}\boldsymbol{v}_{1}=\boldsymbol{v}_{m}^{N} \mathrm{e}^{-\mathrm{i} k_{m}^{N} z}, & \boldsymbol{T}_{1}=\boldsymbol{T}_{m}^{N} \mathrm{e}^{-\mathrm{i} k_{m}^{N} z} \\ \boldsymbol{v}_{2}=\boldsymbol{v}_{n}^{M} \mathrm{e}^{-\mathrm{i} k_{n}^{M} z}, & \boldsymbol{T}_{2}=\boldsymbol{T}_{n}^{M} \mathrm{e}^{-\mathrm{i} k_{n}^{M} z}\end{cases}
$$

After substituting Eq. (4) into Eq. (3), we obtain $-4 \mathrm{i}\left(k_{m}^{N}-k_{n}^{M^{*}}\right) P_{n m}^{M N}=\iint_{D} \nabla_{r \theta} \cdot\left(\boldsymbol{v}_{n}^{M^{*}} \cdot \boldsymbol{T}_{m}^{N}+\boldsymbol{v}_{m}^{N} \cdot \boldsymbol{T}_{n}^{M^{*}}\right) \mathrm{d} \sigma$,

where $D$ is the cross section and $P_{n m}^{M N}$ is defined as

$$
P_{n m}^{M N}=-\frac{1}{4} \iint_{D}\left(\boldsymbol{v}_{n}^{M^{*}} \cdot \boldsymbol{T}_{m}^{N}+\boldsymbol{v}_{m}^{N} \cdot \boldsymbol{T}_{n}^{M^{*}}\right) \cdot \boldsymbol{e}_{z} \mathrm{~d} \sigma .
$$

For two different waveguide modes, the orthogonality relation can then be written as

$$
P_{n m}^{M N}=0, \quad \text { unless } M=N \text { and } k_{n}^{M}=k_{m}^{N} .
$$

\subsection{NME amplitude}

In the complex reciprocity relation equation (3), let $\boldsymbol{v}_{1}$ represents the actual particle velocity field in the cylinder and $\boldsymbol{v}_{2}$ denotes the particle velocity field of a normal mode, which is given as

$$
\left\{\begin{array}{l}
\boldsymbol{v}_{1}=\boldsymbol{v}=\sum_{N=0}^{\infty} \sum_{m} A_{m}^{N}(z) \boldsymbol{v}_{m}^{N}(r, \theta), \\
\boldsymbol{v}_{2}=\boldsymbol{v}_{n}^{M}(r, \theta) \mathrm{e}^{-\mathrm{i} k_{n}^{M} z}
\end{array}\right.
$$

Using Eqs. (3), (7), and (8), after some intermediary transformations, one obtains

$$
\begin{aligned}
& 4 P_{m m}^{N N}\left(\frac{\mathrm{d}}{\mathrm{d} z}+\mathrm{i} k_{m}^{N}\right) A_{m}^{N}(z) \\
& =\oint_{\partial_{1} D} \boldsymbol{v}_{m}^{N^{*}} \cdot\left(\boldsymbol{T} \cdot \boldsymbol{n}_{1}\right) \mathrm{d} s+\oint_{\partial_{2} D} \boldsymbol{v}_{m}^{N^{*}} \cdot\left(\boldsymbol{T} \cdot \boldsymbol{n}_{2}\right) \mathrm{d} s,
\end{aligned}
$$

Table 1 Dimensions and material properties of a steel pipe

\begin{tabular}{cccccc}
\hline \multicolumn{2}{c}{ Dimension } & & \multicolumn{3}{c}{ Material property } \\
\cline { 1 - 2 } \cline { 5 - 6 } Outer diameter, $D_{\mathrm{o}}(\mathrm{mm})$ & Thickness, $h(\mathrm{~mm})$ & & Density, $\rho\left(\mathrm{kg} / \mathrm{m}^{3}\right)$ & Young's modulus, $E(\mathrm{GPa})$ & Poisson's ratio, $v$ \\
\hline 140 & 4.5 & 7800 & 210 & 0.28 \\
\hline
\end{tabular}


where $\boldsymbol{T} \cdot \boldsymbol{n}_{1}$ and $\boldsymbol{T} \cdot \boldsymbol{n}_{2}$ are the loading conditions on the inner and outer boundaries, $\partial_{1} D$ and $\partial_{2} D$, respectively, of the cylinder. Considering the traction-free condition on the inner and outer boundaries of the hollow cylinder (Fig. 1), Eq. (9) can be integrated with the result, that is, the general form of the forward normal mode amplitude:

$$
\begin{aligned}
A_{+m}^{N}(z)= & \frac{\mathrm{e}^{-\mathrm{i} k_{m}^{N} z}}{4 P_{m m}^{N N}} \int_{c}^{z} \mathrm{e}^{\mathrm{i} k_{m}^{N} \xi} \\
& \cdot\left[\oint_{\partial_{1} D} \boldsymbol{v}_{m}^{N^{*}} \cdot\left(\boldsymbol{T} \cdot \boldsymbol{n}_{1}\right) \mathrm{d} s\right. \\
& \left.+\oint_{\partial_{2} D} \boldsymbol{v}_{m}^{N^{*}} \cdot\left(\boldsymbol{T} \cdot \boldsymbol{n}_{2}\right) \mathrm{d} s\right] \mathrm{d} \xi .
\end{aligned}
$$

Only source condition on the outer boundary will be considered in this study.

\section{Modeling of magnetostrictive transducers}

Typical magnetostrictive transducers are modeled in this study (Hirao and Ogi, 2003; Ribichini et al., 2010; 2011; 2012). Ferromagnetic materials have the property that when placed in a magnetic field, they are mechanically deformed. This property is called the magnetostrictive effect. The reverse phenomenon in which the magnetic induction of the material changes when it is mechanically deformed is called inverse magnetostrictive effect. The magnetostrictive phenomenon and its inverse effect can be utilized for the generation and detection of ultrasonic guided waves. Magnetostrictive guided wave transducers, including longitudinal wave transducer based on Joule effect and its inverse effect and torsional wave transducer based on Wiedemann effect and its inverse effect, can produce axial and circumferential loading, respectively.

The magnetostrictive effect relating the magnetic and mechanical material states can be assumed to take a similar form as that for the piezoelectricity (Hirao and Ogi, 2003; Ribichini et al., 2010):

$$
\left\{\begin{array}{l}
\boldsymbol{S}=\boldsymbol{s} \boldsymbol{T}+\boldsymbol{d} \boldsymbol{H}, \\
\boldsymbol{B}=\boldsymbol{d}^{\mathrm{T}} \boldsymbol{T}+\boldsymbol{\mu H},
\end{array}\right.
$$

where $\boldsymbol{S}$ and $\boldsymbol{T}$ are strain and stress, while $\boldsymbol{H}$ and $\boldsymbol{B}$ are magnetic field and magnetic induction, respectively, $\boldsymbol{s}$ is the elastic compliance matrix, $\boldsymbol{\mu}$ is the magnetic permeability matrix, and $\boldsymbol{d}=\partial \boldsymbol{S} / \partial \boldsymbol{H}$ is the piezomagnetic coupling matrix.

Eq. (11) assumes a linear $\boldsymbol{S}-\boldsymbol{H}$ relation, but it is experimentally known that ferromagnetic materials exhibit a highly nonlinear magnetostriction curve. In the case of magnetostrictive guided wave transducers, a small dynamic magnetic field $\tilde{\boldsymbol{H}}$ is superimposed on a much larger static bias magnetic field $\overline{\boldsymbol{H}}$; therefore, the linear assumption is locally valid. When the small dynamic magnetic field $\tilde{\boldsymbol{H}}$ is superimposed on a larger static bias magnetic field $\overline{\boldsymbol{H}}$, the resulting strain can be decomposed into a static component $\overline{\boldsymbol{S}}$ and a dynamic component $\tilde{\boldsymbol{S}}$ (Ribichini et al., 2010):

$$
\left\{\begin{array}{l}
\boldsymbol{H}=\overline{\boldsymbol{H}}+\tilde{\boldsymbol{H}}, \\
\boldsymbol{S}=\overline{\boldsymbol{S}}+\tilde{\boldsymbol{S}} .
\end{array}\right.
$$

Here only the dynamic components of the constitutive equations are considered:

$$
\left\{\begin{array}{l}
\tilde{\boldsymbol{S}}=\boldsymbol{s} \tilde{\boldsymbol{T}}+\boldsymbol{d} \tilde{\boldsymbol{H}} \\
\boldsymbol{B}=\boldsymbol{d}^{\mathrm{T}} \tilde{\boldsymbol{T}}+\boldsymbol{\mu} \tilde{\boldsymbol{H}},
\end{array}\right.
$$

where the dynamic magnetostriction matrix $\boldsymbol{d}$ depends on the direction of the magnetization. Note that the linearized equations are valid in the large static bias magnetic field assumption, even when static component of linear constitutive equations is invalid. The first equation of Eq. (13) accounts for the direct magnetostrictive effect used in the generation of elastic waves, while the second equation describes the inverse magnetostriction used in the detection process.

Both the static bias magnetic field $H_{0 z}$ and small dynamic magnetic field $H_{z}$ of longitudinal wave transducers are applied along the $z$ direction, as is shown in Fig. 3. When $H_{z}$ is much smaller than $H_{0 z}$, the coupling matrix can be approximated as 


$$
\boldsymbol{d}=\left[\begin{array}{cccccc}
0 & 0 & 0 & 0 & \frac{3 S_{\mathrm{t}}}{H_{0 z}} & 0 \\
0 & 0 & 0 & \frac{3 S_{\mathrm{t}}}{H_{0 z}} & 0 & 0 \\
-\frac{1}{2} \gamma & -\frac{1}{2} \gamma & \gamma & 0 & 0 & 0
\end{array}\right]^{\mathrm{T}}
$$

where $\gamma=\partial S_{3} / \partial H_{z}$ is the slope of magnetostriction curve and $S_{\mathrm{t}}$ is the total magnetostrictive strain. Note that the $S_{3}$ strain component is solely responsible for the generation of longitudinal waves, so the constitutive equations for longitudinal wave transducers can be simplified and given as

$$
\left\{\begin{array}{l}
\tilde{S}_{3}=s_{33} \tilde{T}_{3}+d_{33} \tilde{H}_{z}, \\
B_{z}=d_{33} \tilde{\sigma}_{3}+\mu_{3} \tilde{H}_{z}
\end{array}\right.
$$

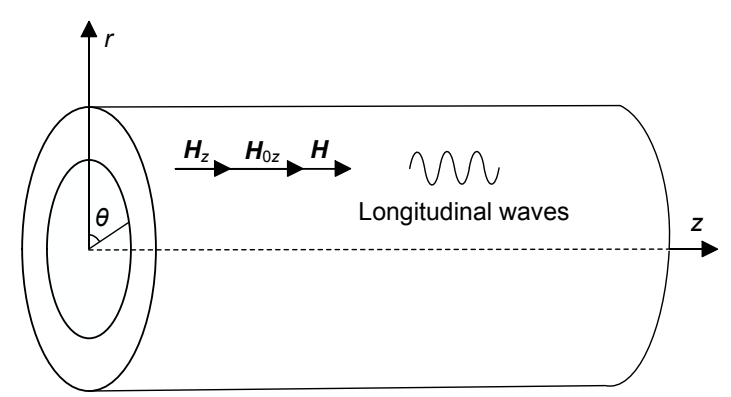

Fig. 3 Schematic of longitudinal wave transducers on the outer surface of a pipe

For torsional wave transducers, the static bias magnetic field is applied along the $\theta$ direction, while the small dynamic magnetic field is applied along the $z$ direction, as is shown in Fig. 4. Under the large static bias magnetic field assumption, the coupling matrix for torsional wave transducers can also be approximated as

$$
\boldsymbol{d}=\left[\begin{array}{cccccc}
0 & 0 & 0 & 0 & 0 & \frac{3 S_{\mathrm{t}}}{H_{0 \theta}} \\
-\frac{1}{2} \gamma & \gamma & -\frac{1}{2} \gamma & 0 & 0 & 0 \\
0 & 0 & 0 & \frac{3 S_{\mathrm{t}}}{H_{0 \theta}} & 0 & 0
\end{array}\right]^{\mathrm{T}} .
$$

Since only the $S_{4}$ shear strain component is responsible for generation of torsional waves, the constitutive equations for torsional wave transducers can also be simplified as

$$
\left\{\begin{array}{l}
\tilde{S}_{4}=s_{44} \tilde{T}_{4}+d_{43} \tilde{H}_{z} \\
B_{z}=d_{43} \tilde{T}_{3}+\mu_{3} \tilde{H}_{z}
\end{array}\right.
$$

Note that thin magnetostrictive patches such as nickel and ferrocobalt strips are often used to generate and measure elastic waves. The magnetostrictive longitudinal and torsional wave transducers described above can be modeled using commercial finite element software packages, such as COMSOL Multiphysics.

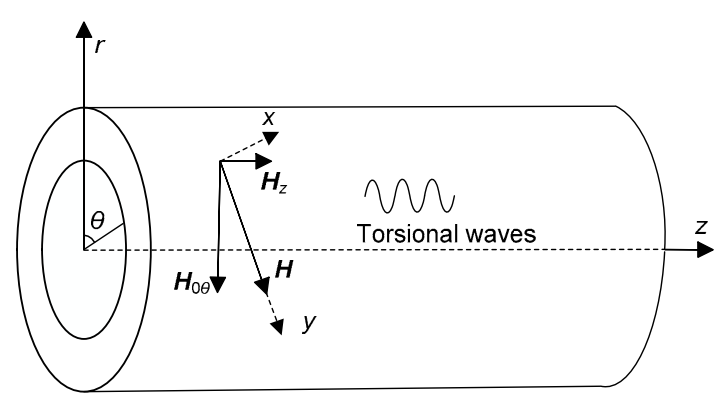

Fig. 4 Schematic of torsional wave transducers on the outer surface of a pipe

\section{Perturbation analysis of cylinders under magnetostrictive loadings}

As described in Section 3, magnetostrictive transducers are capable of providing circumferential and axial loadings $\left(\boldsymbol{T}=\boldsymbol{s}^{-1}(\boldsymbol{S}-\boldsymbol{d H})\right)$ on the surface of a hollow cylinder. Therefore, only circumferential and axial magnetostrictive loadings on the outer boundary of the cylinder are considered in this study, which can be written as

$$
\begin{aligned}
& \boldsymbol{T} \cdot \boldsymbol{n}_{1}=0, \\
& \boldsymbol{T} \cdot \boldsymbol{n}_{2}= \begin{cases}-p_{1}(\theta) p_{2}(z) \boldsymbol{e}_{\theta / z}, & |z| \leq L,|\theta| \leq \alpha, r=R_{\mathrm{o}}, \\
0, & |z|>L,|\theta|>\alpha, r=R_{\mathrm{o}},\end{cases}
\end{aligned}
$$

where $p_{1}(\theta)$ and $p_{2}(z)$ are circumferential and axial loading functions, and $L$ and $\alpha$ are axial and circumferential extent of the loading area, respectively. 
Substituting Eqs. (18) and (19) into Eq. (10) gives

$$
\begin{gathered}
A_{+m}^{N}(z)=\frac{R_{m \gamma}^{N}(b) \mathrm{e}^{-\mathrm{i} k_{m}^{N} z}}{4 P_{m m}^{N N}}\left\langle\Theta_{\gamma}^{N}, p_{1}(\theta)\right\rangle \cdot\left\langle p_{2}(z), \mathrm{e}^{\mathrm{i} k_{m}^{N} z}\right\rangle, \\
z \geq L(\gamma=\theta, z),
\end{gathered}
$$

where $\left\langle\Theta_{\gamma}^{N}, p_{1}(\theta)\right\rangle$ and $\left\langle p_{2}(z), \mathrm{e}^{\mathrm{i} k_{m}^{N} z}\right\rangle$ are the circumferential and axial amplitude factors, respectively, which are defined as

$$
\begin{gathered}
\left\langle\Theta_{\gamma}^{N}, p_{1}(\theta)\right\rangle=H_{\theta}=b \int_{\beta}^{\beta+2 \pi} \Theta_{\gamma}^{N}(N \theta) p_{1}(\theta) \mathrm{d} \theta, \\
\left\langle p_{2}(z), \mathrm{e}^{\mathrm{i} k_{m}^{N} z}\right\rangle=H_{z}=\int_{-\infty}^{\infty} p_{2}(z) \mathrm{e}^{\mathrm{i} k_{m}^{N} z} \mathrm{~d} z
\end{gathered}
$$

\subsection{Magnetostrictive partial loading}

A magnetostrictive partial loading is nonaxisymmetric and only covers a portion of the pipe surface over some circumferential angle, as is shown in Fig. 5. The loading distribution functions $p_{1}(\theta)$ and $p_{2}(z)$ are given as (Shin and Rose, 1999)

$$
\begin{aligned}
& p_{1}(\theta)= \begin{cases}P_{1}, & |\theta| \leq \alpha, \\
0, & |\theta|>\alpha,\end{cases} \\
& p_{2}(z)= \begin{cases}P_{2}, & |z| \leq L, \\
0, & |z|>L .\end{cases}
\end{aligned}
$$

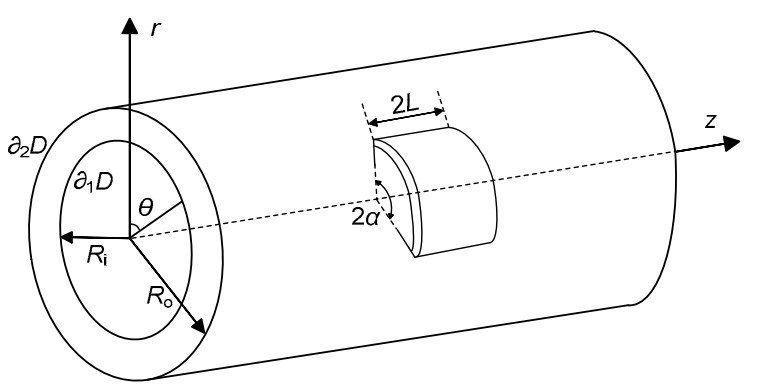

Fig. 5 A hollow cylinder with partial loading of a transducer with $2 L$ width and $2 \alpha$ circumferential coverage angle

Substituting Eqs. (23) and (24) into Eqs. (21) and (22), the source functions can be obtained:

$$
\begin{gathered}
H_{\theta}= \begin{cases}b P_{1}(2 \alpha), & N=0, \\
2 b P_{1} \frac{\sin (N \alpha)}{N}, & N \geq 1,\end{cases} \\
H_{z}=\frac{2 P_{2} \sin \left(k_{m}^{N} L\right)}{k_{m}^{N}} .
\end{gathered}
$$

According to Eq. (25), the circumferential amplitude factor $H_{\theta}$ is a function of circumferential order $N$ and loading angle $\alpha$, but is independent of phase velocity and frequency. There is a linear relation between the axisymmetric circumferential amplitude factor and loading angle $\alpha$, while the nonaxisymmetric circumferential amplitude factor follows a sinusoidal trend as a function of the loading angle $\alpha$ for a certain circumferential order $N$. Also, $H_{\theta}=0(N \geq 1)$ when $2 \alpha=2 \pi$, which indicates that only axisymmetric modes will be excited if the magnetostrictive load is axisymmetric. If $N \alpha=p \pi, p=0,1$, $2, \ldots$, then $H_{\theta}=0(N \geq 1)$, which implies that normal modes with circumferential order $N$ will not be excited. The relative circumferential amplitude factors for different loading angles of $45^{\circ}, 90^{\circ}, 180^{\circ}$, and $360^{\circ}$ are shown in Fig. 6 . It is evident that the amplitude of non-axisymmetric flexural modes decreases, while the loading angles of magnetostrictive partial loading increase. The flexural modes are completely suppressed when the loading angle approaches $360^{\circ}$.

According to Eq. (26), the axial amplitude factor $H_{z}$ is a function of loading length $2 L$ and wavenumber $k_{m}^{N}$ of mode with circumferential order $N$ in the $m$ th family. Furthermore, $H_{z}$ will reach the maximum when $k_{m}^{N} L=(2 p+1) \pi / 2, p=0,1,2, \ldots$; thus, $2 L=(2 p+1) \lambda_{m}^{N} / 2, p=0,1,2, \ldots$, are efficient loading lengths for a mode at a particular frequency. The axial amplitude factor can be rewritten as a function of frequency $f$, phase velocity $C p_{m}^{N}$, and loading length $2 L$ such that

$$
H_{z}=\frac{2 P_{2} \sin \left[\left(2 \pi f /\left(C p_{m}^{N}\right)\right) L\right]}{2 \pi f /\left(C p_{m}^{N}\right)} .
$$

The phase velocity spectra $(f=32 \mathrm{kHz})$ of $H_{z}$ with different loading lengths $2 L$ are shown in Fig. 7. It can be observed that $H_{z}$ has a very broad phase 

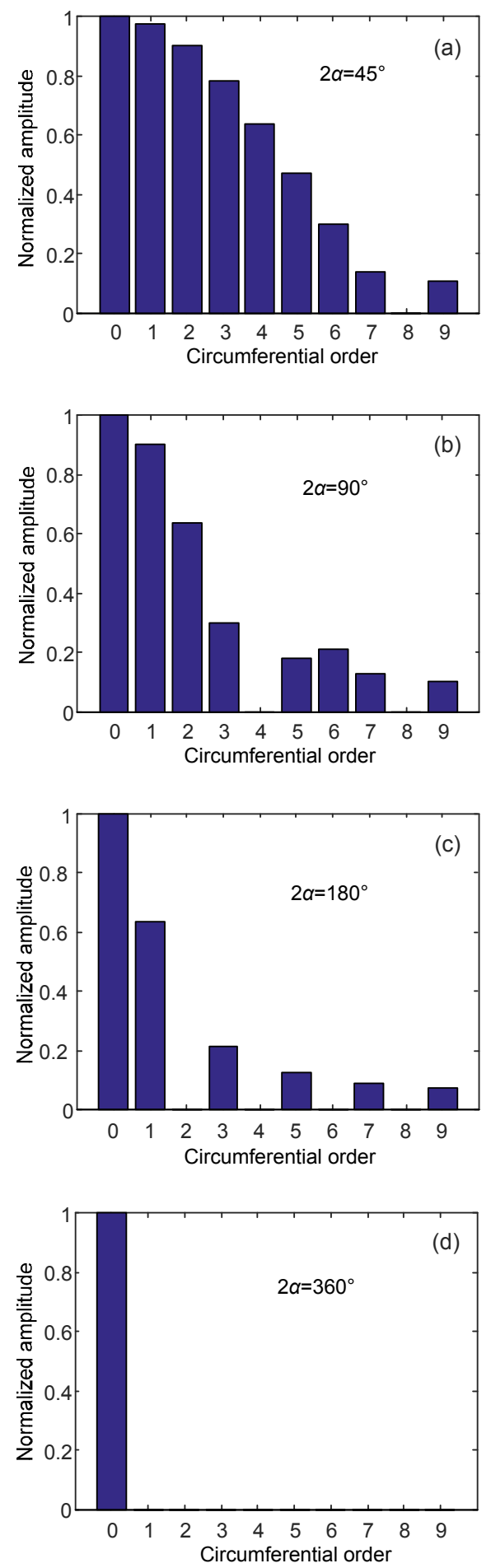

Fig. 6 Relative circumferential amplitude factors for axisymmetric and non-axisymmetric modes at loading angles of $2 \alpha=45^{\circ}$ (a), $90^{\circ}$ (b), $180^{\circ}$ (c), and $360^{\circ}$ (d)

velocity bandwidth, which means it has poor phase velocity selectivity. The frequency spectra of $H_{z}$ for
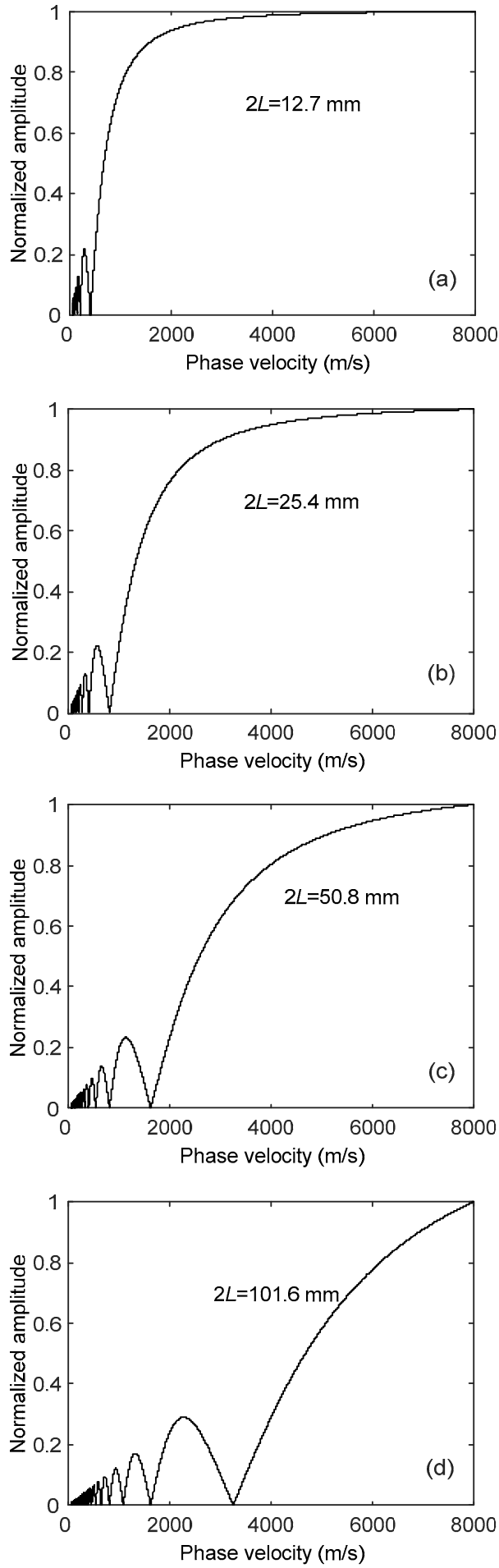

Fig. 7 Phase velocity spectra of the axial amplitude factor at different parameters: (a) $2 L=12.7 \mathrm{~mm}$; (b) $2 L=$ $25.4 \mathrm{~mm}$; (c) $2 L=50.8 \mathrm{~mm}$; (d) $2 L=101.6 \mathrm{~mm}$

axisymmetric modes $T(0,1)$ and $L(0,2)$ are shown in Fig. 8, which indicates that the axial amplitude 
factor has different frequency sensitivities for different loading lengths with the extreme point corresponding to the condition $2 L=(2 p+1) \lambda_{m}^{N} / 2, p=0$, $1,2, \ldots$
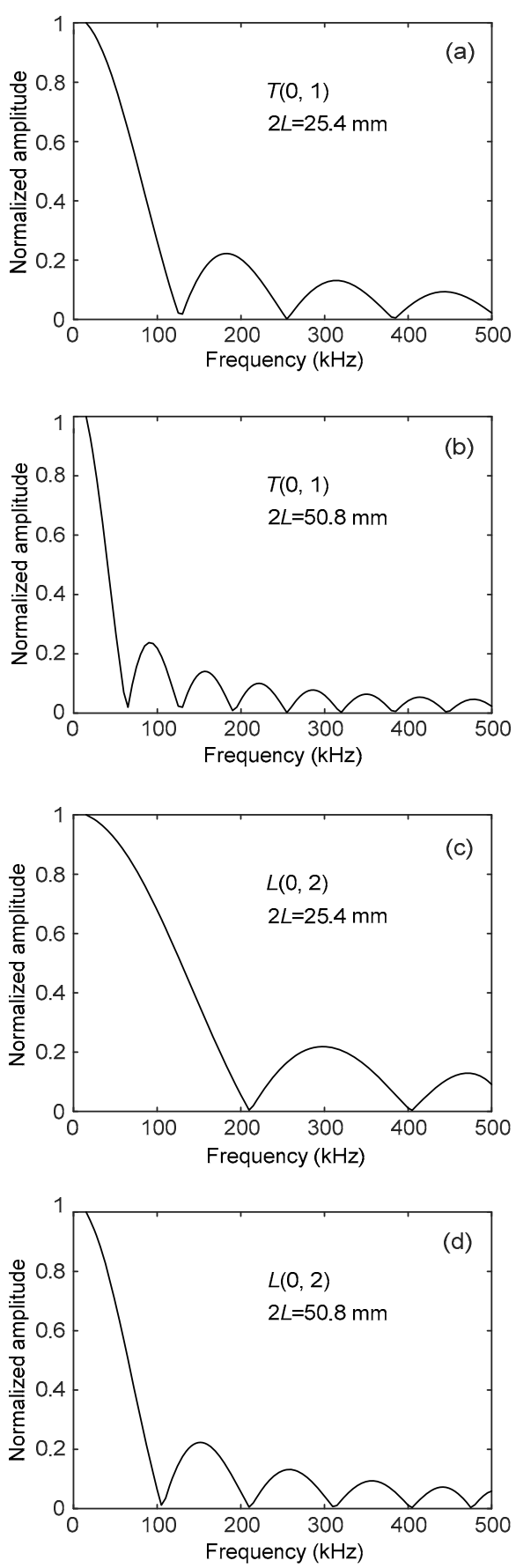

Fig. 8 Frequency spectra of the axial amplitude factor at different parameters: (a) $T(0,1), 2 L=25.4 \mathrm{~mm}$; (b) $T(0$, 1), $2 L=50.8 \mathrm{~mm}$; (c) $L(0,2), 2 L=25.4 \mathrm{~mm}$; (d) $L(0,2)$, $2 L=50.8 \mathrm{~mm}$
Substituting Eqs. (25) and (26) into Eq. (20), the amplitude of normal mode with circumferential order $N$ in the $m$ th family of an elastic hollow cylinder under magnetostrictive partial loading is obtained. A sample calculation of amplitude factor is carried out and shown in Fig. 9 for $T(0,1)$ and $F(N$, 2) modes generated by a $45^{\circ}$ circumferential loading at $128 \mathrm{kHz}$ in an elastic pipe with the parameters given in Table 1.

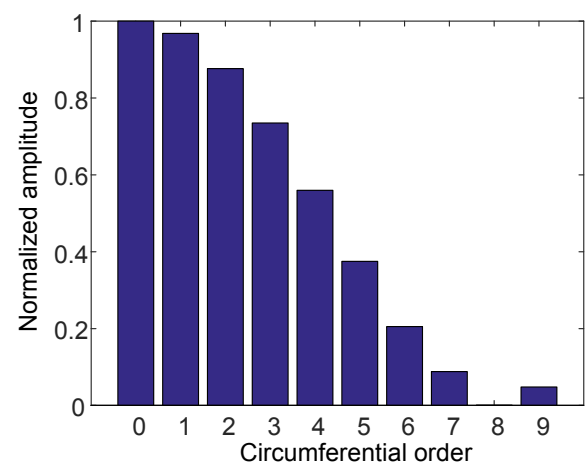

Fig. 9 Amplitude factors for $T(0,1)$ and $F(N, 2)$ modes generated by $\mathrm{a}^{\circ}$ magnetostrictive circumferential loading at $128 \mathrm{kHz}$ in an elastic pipe

\subsection{Magnetostrictive axial array loading}

Consider the case of axial array loading in a comb transducer (Ditri et al., 1993; Rose et al., 1998; Hay and Rose, 2002; Philtron and Rose, 2014), as is shown in Fig. 10, where $\eta$ magnetostrictive elements are equally spaced along the axial direction of a cylinder. Here, $2 \beta$ is the element width and $2 \delta$ is the spacing. The loading distribution functions $p_{1}(\theta)$ and $p_{2}(z)$ (without time delay) can be written as

$$
\begin{aligned}
& p_{1}(\theta)=P_{1}, \quad \theta \in[0,2 \pi), \\
& p_{2}(z)= \begin{cases}P_{2}, & z \in G, \\
0, & z \notin G,\end{cases}
\end{aligned}
$$

where

$$
\begin{gathered}
G=\bigcup_{\xi=1}^{\eta / 2} g_{\xi}, \\
g_{\xi} \equiv\{z:(2 \xi-1) \delta+2(\xi-1) \beta \leq|z| \leq(2 \xi-1) \delta+2 \xi \beta\} .
\end{gathered}
$$

Substituting Eqs. (21) and (22) into Eqs. (14) and (15), the source functions are obtained as: 


$$
\begin{gathered}
H_{\theta}= \begin{cases}2 \pi b P_{1}, & N=0, \\
0, & N \geq 1,\end{cases} \\
H_{z}=\sum_{\xi=1}^{\eta / 2} \int_{g_{\xi}} P_{2} \mathrm{e}^{\mathrm{i} k_{m}^{N} z} \mathrm{~d} z=P_{2} \frac{\sin \left(k_{m}^{N} \beta\right)}{\beta} \\
\times \frac{\cos \left(k_{m}^{N}[(\eta-1) D]\right)-\cos \left(k_{m}^{N}[(\eta+1) D]\right)}{1-\cos \left(2 k_{m}^{N} D\right)},
\end{gathered}
$$

where the element distance $2 D=2 \beta+2 \delta$.

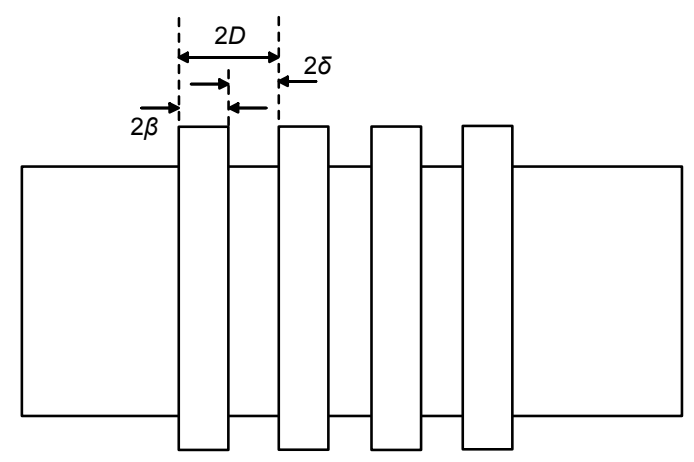

Fig. 10 Schematic of elastic hollow cylinders under magnetostrictive axial array loading

According to Eq. (31), the axial amplitude factor depends on the element width $2 \beta\left(H_{\beta}\right)$, the element distance $2 D$, and the number of elements $\eta$ $\left(H_{D}\right)$. The extremum conditions of $H_{\beta}$ and $H_{D}$ are $\tan \left(k_{m}^{N} \beta\right)=k_{m}^{N} \beta \quad$ and $\quad \eta \cdot \tan \left(k_{m}^{N} D\right)=\tan \left(\eta \cdot k_{m}^{N} D\right)$, respectively, where $k_{m}^{N}=p \pi / D, p=0,1,2, \ldots$, and $\lambda_{m}^{N}=2 D / p, p=0,1,2, \ldots$

The phase velocity spectra $(f=64 \mathrm{kHz})$ of axial amplitude factor at different values of $2 \beta, 2 D$, and $\eta$ are shown in Fig. 11. It is obvious that magnetostrictive axial array loading has good phase velocity selectivity. Furthermore, it can be seen from Figs. 11a and $11 \mathrm{~b}$ that the center phase velocity is mainly dependent on element distance $2 D$; a larger element distance corresponds to a higher phase velocity. From Figs. 11a and 11c, a conclusion can be drawn that the phase velocity bandwidth is mainly dependent on the number of elements $\eta$. Moreover, it can be seen from Figs. $11 \mathrm{~b}$ and $11 \mathrm{~d}$ that the element width $2 \beta$ makes a contribution to the amplitude of $H_{z}$ at the center phase velocity.
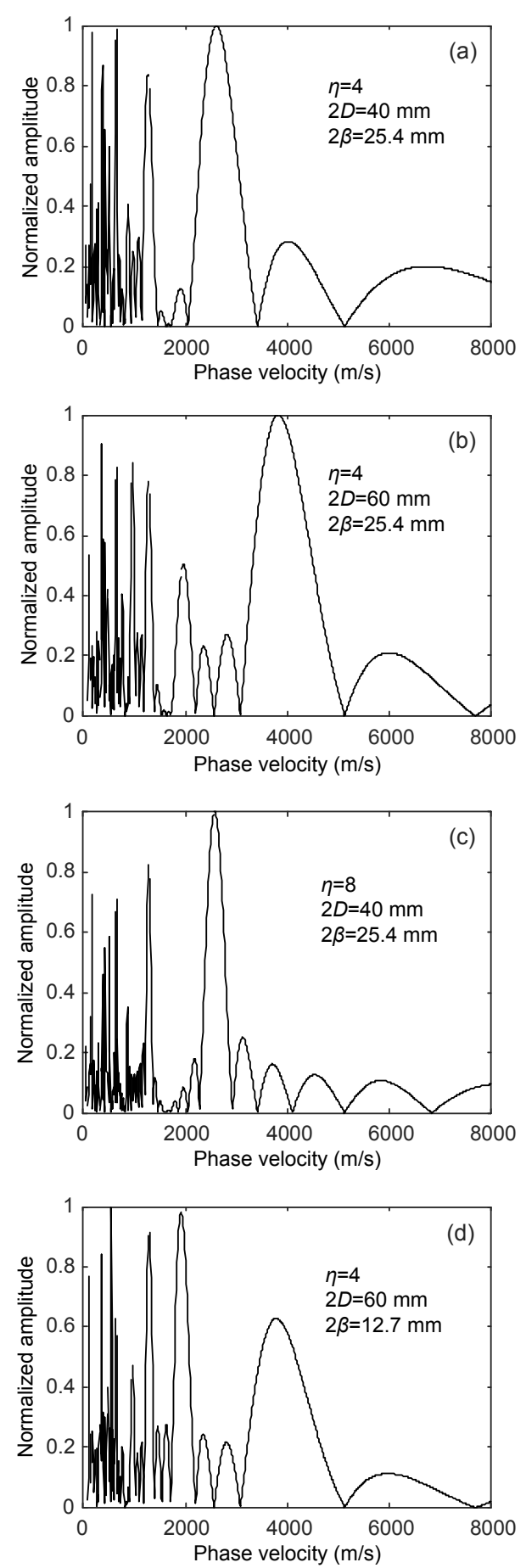

Fig. 11 Phase velocity spectra of axial amplitude factor for axial array loading at different parameters: (a) $\eta=4$, $2 D=40 \mathrm{~mm}, 2 \beta=25.4 \mathrm{~mm}$; (b) $\eta=4,2 D=60 \mathrm{~mm}, 2 \beta=$ $25.4 \mathrm{~mm}$; (c) $\eta=8,2 D=40 \mathrm{~mm}, 2 \beta=25.4 \mathrm{~mm}$; (d) $\eta=4,2 D=$ $60 \mathrm{~mm}, 2 \beta=12.7 \mathrm{~mm}$

The frequency spectra of $H_{z}$ for axisymmetric modes $T(0,1)$ and $L(0,2)$ are shown in Fig. 12. The 
influence of array parameters $2 \beta, 2 D$, and $\eta$ on the frequency spectrum is similar to that on the phase velocity spectrum. Furthermore, if time delay $\mathrm{e}^{-\mathrm{i} \omega(\xi) t d)}$ is applied to each element, the comb-type
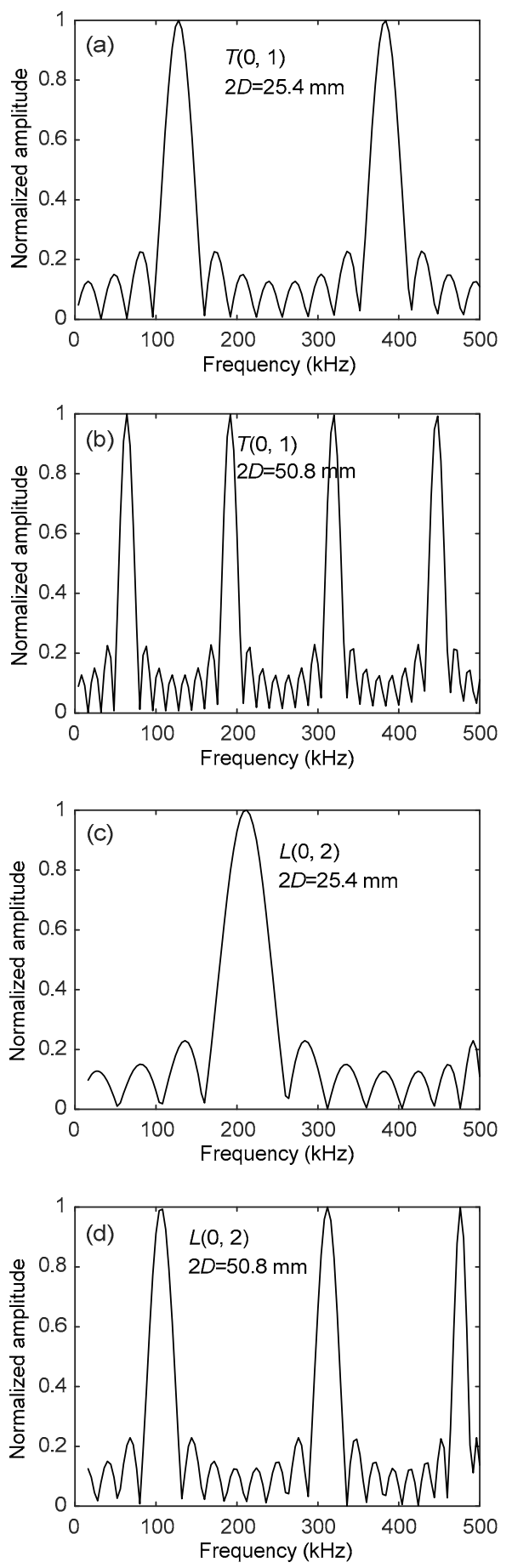

Fig. 12 Frequency spectra of axial amplitude factor for axial array loading at different parameters: (a) $T(0,1)$, $2 D=25.4 \mathrm{~mm}$; (b) $T(0,1), 2 D=50.8 \mathrm{~mm}$; (c) $L(0,2), 2 D=$ $25.4 \mathrm{~mm}$; (d) $L(0,2), 2 D=50.8 \mathrm{~mm}$ magnetostrictive transducer will provide even better phase velocity, frequency selectivity, and guided wave mode controllability.

\subsection{Magnetostrictive circular array loading}

Consider the circular array loading case ( $\mathrm{Li}$ and Rose, 2001b; 2002), as is shown in Fig. 13, where $\eta$ equally sized $(2 L \times 2 \alpha)$ magnetostrictive elements are equally spaced along the circumferential direction of a cylinder with their circumferential position at $\theta=$ $(2 \xi-1) \pi / \eta, \xi=1,2, \ldots, \eta$. The loading distribution functions $p_{1}(\theta)$ and $p_{2}(z)$ can be written as

$$
p_{1}(\theta)= \begin{cases}P_{1}, & \theta \in G, \\ 0, & \theta \notin G,\end{cases}
$$

where

$$
\begin{gathered}
G=\bigcup_{\xi=1}^{\eta} g_{\xi}, \\
g_{\xi} \equiv\left\{\theta: \frac{(2 \xi-1) \pi}{\eta}-\alpha<\theta<\frac{(2 \xi-1) \pi}{\eta}+\alpha\right\}, \\
p_{2}(z)= \begin{cases}P_{2}, & |z| \leq L, \\
0, & \text { otherwise. }\end{cases}
\end{gathered}
$$

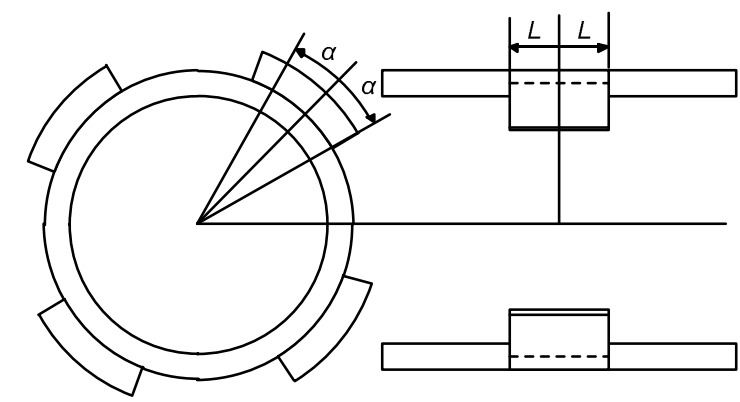

Fig. 13 Schematic of elastic hollow cylinders under magnetostrictive circular array loading

Substituting Eqs. (32) and (33) into Eqs. (21) and (22), the source functions are obtained as follows:

$$
\begin{aligned}
H_{\theta} & = \begin{cases}b P_{1} \eta(2 \alpha), & N=0, \\
2 b P_{1} \frac{\sin (N \alpha)}{N} \sum_{\xi=1}^{\eta} \cos \left(\frac{N(2 \xi-1) \pi}{\eta}\right), & N \geq 1,\end{cases} \\
& = \begin{cases}2 b P_{1} \frac{\sin (p \eta \alpha)}{p}(-1)^{p}, & N=p \eta, \\
0, & N \neq p \eta,\end{cases}
\end{aligned}
$$




$$
H_{z}=\frac{2 P_{2} \sin \left(k_{m}^{N} L\right)}{k_{m}^{N}} .
$$

Eq. (35) is the same as Eq. (26), and thus, the conclusions are not repeated here. According to Eq. (34), only modes with circumferential order $N=p \eta, p=0,1,2, \ldots$ can be excited in a hollow cylinder with a magnetostrictive circular array loading. The relative circumferential amplitude factor with respect to that of axisymmetric modes can be defined as

$$
\left|\frac{H_{\theta}^{N}}{H_{\theta}^{0}}\right|=\frac{\sin (p \eta \alpha)}{p \eta \alpha}=\frac{\sin (p \Delta \pi)}{p \Delta \pi},
$$

where $\Delta=\eta(2 \alpha) / 2 \pi, \Delta \in[0,1]$ is the loading area ratio.

As can be seen from Fig. 14, the relative amplitude of higher harmonics decreases as the loading area ratio $\Delta$ increases and the relative amplitude decreases at a faster rate for higher circumferential orders, which indicates that magnetostrictive circular array loading can be designed to generate a specific circumferential order of the guided wave modes. In addition, beam steering (Wilcox, 2003) and beam focusing (Hayashi et al., 2005; Sun et al., 2005) can be achieved when time delays are applied to the array elements.

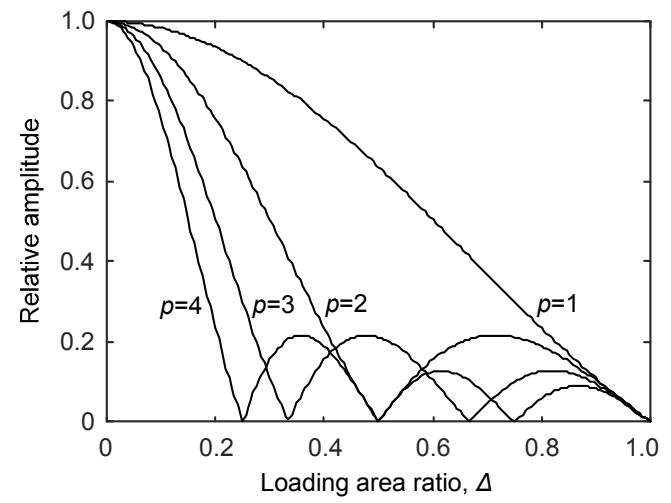

Fig. 14 Relative amplitudes of higher harmonics for circular array loading

\section{Numerical simulation and experiment}

In this section, summarized finite element numerical evaluations (Zhu, 2001; Drozdz, 2008; Mo- reau et al., 2012) and experiments demonstrate the generation of axisymmetric and non-axisymmetric guided wave modes in elastic hollow cylinders under magnetostrictive loadings. A steel pipe, with parameters given in Table 1, is considered in this study with the corresponding phase velocity dispersion curves shown in Fig. 2.

\subsection{Magnetostrictive partial loading}

\subsubsection{Axial partial loading simulation}

The first numerical evaluation involves the axial partial loading at $f=128 \mathrm{kHz}$, with a loading area of axial length $50.8 \mathrm{~mm}$ and a circumferential angle $45^{\circ}$. According to the NME method, the angular profile for $L(0,2)$ and $F(N, 3)$ modes at $1.5 \mathrm{~m}$ from the source was calculated and is shown in Fig. 15. Meanwhile, a model with the same parameters is established and calculated by using the finite element software Abaqus/Explicit. The axial displacements $\left(U_{z}\right)$ of nodes at $z=1.5 \mathrm{~m}$ are recorded, and their axial displacement angular distribution is plotted, which is shown in Fig. 15. The results demonstrated that these two models are in good agreement.
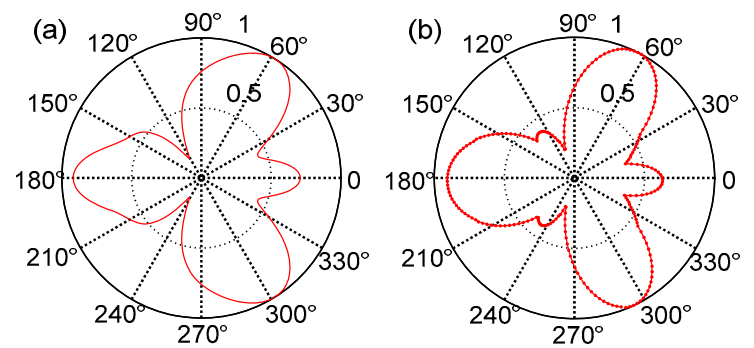

Fig. 15 Angular profiles of $L(0,2)$ and $F(N, 3)$ modes generated by $45^{\circ}$ magnetostrictive axial loading at $128 \mathrm{kHz}$ in an elastic pipe: (a) theoretical prediction; (b) numerical simulation result

\subsubsection{Circumferential partial loading experiment}

The experiment setup is shown in Fig. 16, where a small magnetostrictive transducer with a width of $50.8 \mathrm{~mm}$ is bonded on the surface of the pipe as a transmitter that covers an angle of $45^{\circ}$ along the circumference of the pipe. The central transmitting frequency is $f=250 \mathrm{kHz}$. Thirty-two receivers were placed around the circumference of the pipe at a distance of $1.6 \mathrm{~m}$ from the transmitter. The theoretical and experimental results of angular 
profiles for the $T(0,1)$ and $F(N, 2)$ modes at the frequency of $250 \mathrm{kHz}$ are shown in Fig. 17, which are in good agreement. Since both theoretical and experimental results are in good agreement, the earlier described method involving magnetostrictive partial loading was verified by numerical evaluation and experiment.

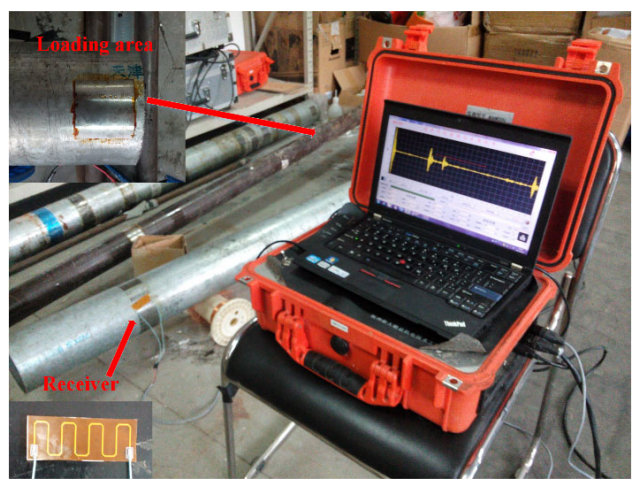

Fig. 16 A setup of circumferential partial loading experiment
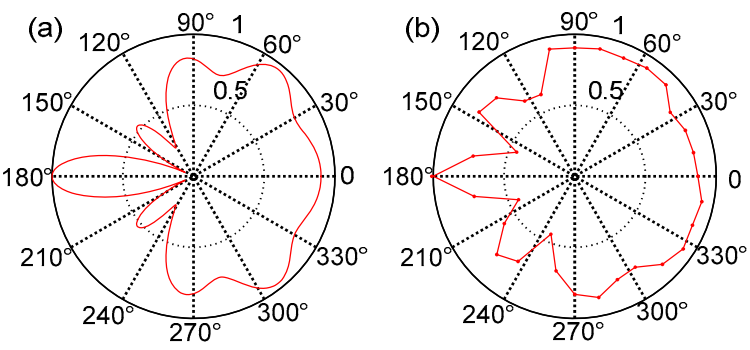

Fig. 17 Angular profiles of $T(0,1)$ and $F(N, 2)$ modes generated by $45^{\circ}$ magnetostrictive circumferential loading at $250 \mathrm{kHz}$ in an elastic pipe: (a) theoretical prediction; (b) experimental result

\subsection{Magnetostrictive axial array loading}

Another numerical evaluation involved an axial array loading at $f=128 \mathrm{kHz}$. Two sets of array parameters were designed for the purpose of $L(0,1)$ excitation and $L(0,2)$ excitation, respectively: (1) $2 \beta=12 \mathrm{~mm}, 2 \delta=4 \mathrm{~mm}$ and (2) $2 \beta=15 \mathrm{~mm}, 2 \delta=6 \mathrm{~mm}$. Finite element models were established and calculated. Fig. 18 shows the snapshot for wave propagation of $L(0,1)$ mode and $L(0,2)$ mode. The $L(0,1)$ mode is dominant on the condition of parameter (1) and $L(0,2)$ mode is dominant on the condition of parameter (2), which demonstrates the mode controllability of magnetostrictive axial array load.
The wave structures of $L(0,1)$ and $L(0,2)$ at $f=128 \mathrm{kHz}$ are shown in Fig. 19. It can be observed that radial displacement $\left(U_{r}\right)$ plays a dominant role for $L(0,1)$ mode, whereas the axial displacement $\left(U_{z}\right)$ for $L(0,2)$ mode at $f=128 \mathrm{kHz}$. Both $r$-component and $z$-component of the displacement field on the external surface of the pipe at the distance of $0.7 \mathrm{~m}$ from the source are recorded and converted into video signals, which are shown in Fig. 20. The above observation is confirmed once again, but it also shows that the modes, especially $L(0,1)$, are not as pure as expected, as radial displacement is dominant for $L(0,1)$ while the external load is in the axial direction. Therefore, radial load, which the magnetostrictive transducer cannot provide, may have the best chance to excite pure $L(0,1)$ mode.

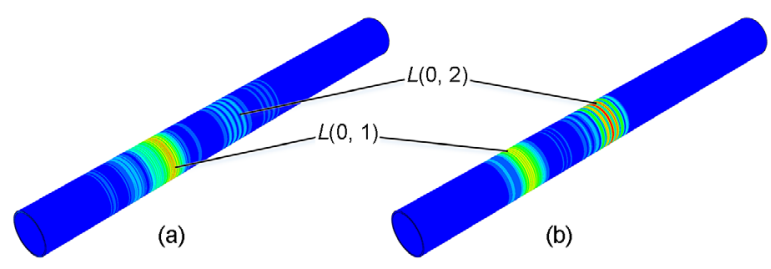

Fig. 18 Snapshot of the wave propagation of $L(0,1)$ (a) and $L(0,2)(b)$ modes in the steel pipes (a) Parameter (1): $2 \beta=12 \mathrm{~mm}, 2 \delta=4 \mathrm{~mm}$; (b) Parameter (2): $2 \beta=15 \mathrm{~mm}, 2 \delta=6 \mathrm{~mm}$
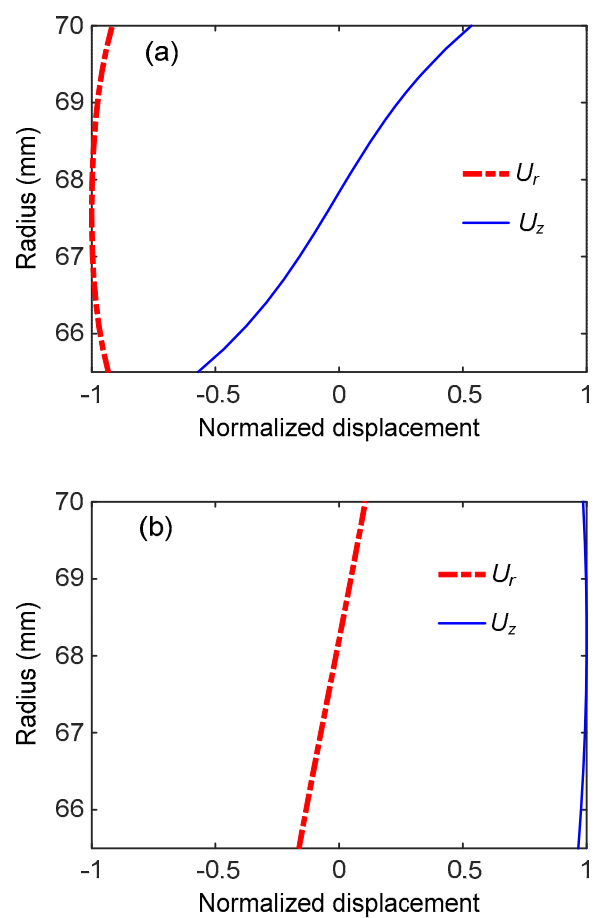

Fig. 19 Wave structure of $L(0,1)$ (a) and $L(0,2)$ (b) modes at $128 \mathrm{kHz}$ in the steel pipe 

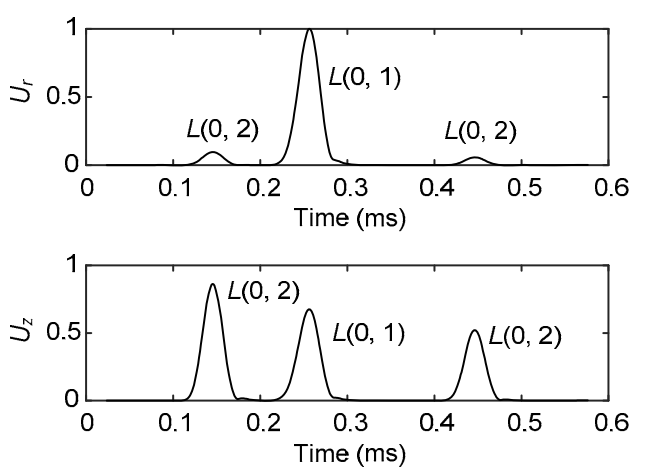

(a)
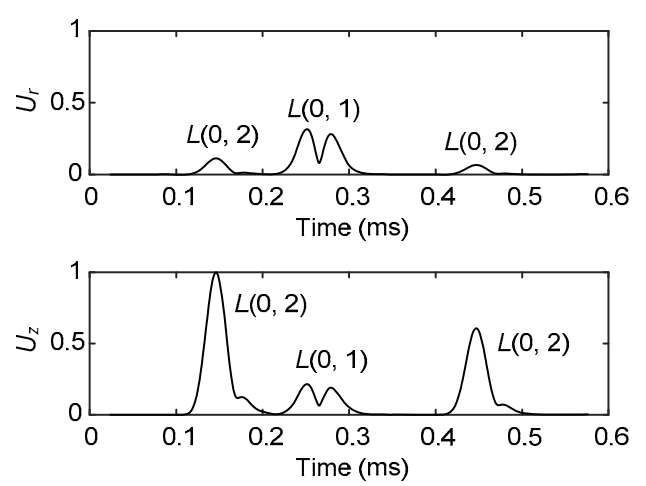

(b)

Fig. 20 Video signals extracted from the excitation of $L(0,1)$ and $L(0,2)$ modes

(a) Parameter (1): $2 \beta=12 \mathrm{~mm}, 2 \delta=4 \mathrm{~mm}$; (b) Parameter (2): $2 \beta=15 \mathrm{~mm}, 2 \delta=6 \mathrm{~mm}$

\subsection{Magnetostrictive circular array loading}

\subsubsection{Circular array axial load simulation}

The last numerical evaluation involves circular array axial load at $f=64 \mathrm{kHz}$, where the number of elements $\eta=2$ and the loading area ratio $\Delta=0.5$. The finite element model is established and used for calculation. Displacements of all the nodes at the distance of $1.5 \mathrm{~m}$ from the source are recorded. The received signals form a $2 \mathrm{D}$ time-circumference data matrix. A Fourier transformation is performed on the data matrix in the circumferential direction, and the circumferential order of the excited modes is extracted. Fig. 21 shows that only modes with circumferential order $N=2 p, p=1,2, \ldots$ are excited, which is in accordance with the theoretical prediction.

\subsubsection{Circular array circumferential load experiment}

A two-element magnetostrictive transducer is bonded around the circumference of the pipe with the loading area ratio $\Delta=0.5$ and the excitation fre- quency $f=250 \mathrm{kHz}$. Thirty-two receivers are placed around the circumference of the pipe at a distance of $1.6 \mathrm{~m}$ from the transmitter. The received signals from 32 receivers form a 2D data matrix. A 2D Fourier transformation is performed on the data matrix, and the circumferential order of excited modes is extracted. Fig. 22 verifies the theoretical prediction once again.

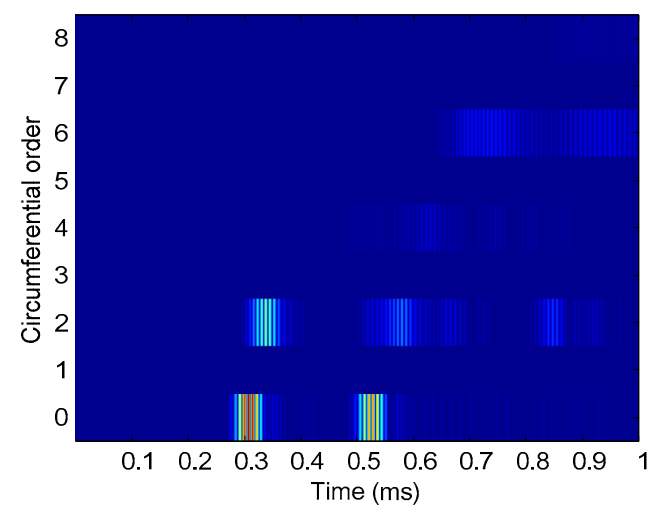

Fig. 21 Circumferential orders of excited modes for circular array axial load numerical simulation

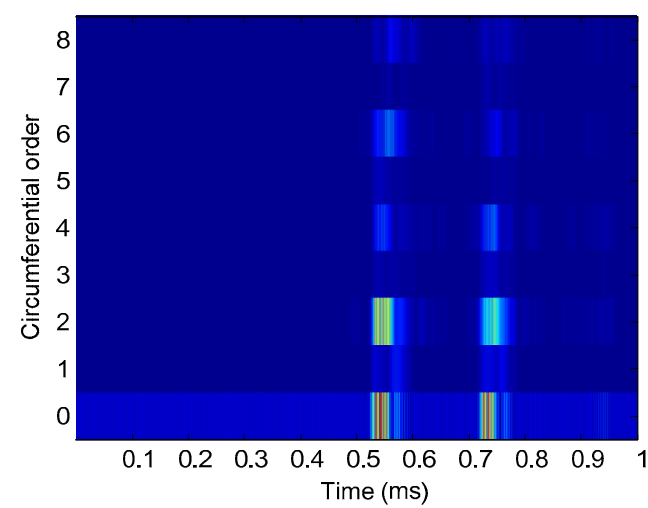

Fig. 22 Circumferential orders of excited modes for circular array circumferential load experiment

\section{Conclusions}

This paper has adopted and developed the classical NME method to analyze forced response and perturbation analysis of a hollow cylinder considering the finite size magnetostrictive loadings, including partial loading, axial array loading, and circular array loading, with the unique feature of magnetostriction taken into consideration. The phase velocity and frequency spectra of guided wave amplitude 
excited by magnetostrictive loading are analyzed in detail, and some basic conclusions have been reached.

Some novel results of a comprehensive perturbation analysis on elastic hollow cylinders under magnetostrictive loadings are summarized as follows. Magnetostrictive partial loading is able to excite guided wave modes of the same family, but has poor phase velocity and frequency selectivity. Axisymmetric loading excites axisymmetric modes only. Angular profiles in a hollow cylinder are sensitive to the circumferential loading length. Magnetostrictive axial array loading has good phase velocity and frequency selectivity and guided wave mode controllability. According to phase velocity and frequency spectra, center phase velocity and center frequency are mainly determined by element distance. Magnetostrictive circular array load only excites guided wave modes whose circumferential order is integer multiple of the number of elements.

\section{References}

Alleyne, D.N., Cawley, P., 1996. The excitation of Lamb waves in pipes using dry-coupled piezoelectric transducers. Journal of Nondestructive Evaluation, 15(1): 11-20. http://dx.doi.org/10.1007/BF00733822

Auld, B.A., 1990. Acoustic Fields and Waves in Solids, Vol. 2. Krieger Publishing Company, Malabar, Florida, USA.

Cho, S.H., Han, S.W., Park, C., et al., 2006. Noncontact torsional wave transduction in a rotating shaft using oblique magnetostrictive strips. Journal of Applied Physics, 100(10): 104903. http://dx.doi.org/10.1063/1.2386942

Ditri, J.J., Rose, J.L., 1992. Excitation of guided elastic wave modes in hollow cylinders by applied surface tractions. Journal of Applied Physics, 72(7):2589-2597. http://dx.doi.org/10.1063/1.351558

Ditri, J.J., Rose, J.L., Pilarski, A., 1993. Generation of guided waves in hollow cylinders by wedge and comb type transducers. In: Thompson, D.O., Chimenti, D.E. (Eds.), Review of Progress in Quantitative Nondestructive Evaluation. Springer US, New York, 12:211-218. http://dx.doi.org/10.1007/978-1-4615-2848-7 26

Drozdz, M.B., 2008. Efficient Finite Element Modeling of Ultrasonic Wave in Elastic Media. PhD Thesis, Imperial College London, London, UK.

Gazis, D.C., 1959. Three dimensional investigation of the propagation of waves in hollow circular cylinders. I. Analytical foundation. The Journal of the Acoustical Society of America, 31(5):568-573. http://dx.doi.org/10.1121/1.1907753

Hay, T.R., Rose, J.L., 2002. Flexible PVDF comb transducers for excitation of axisymmetric guided waves in pipe.
Sensors and Actuators A: Physical, 100(1):18-23. http://dx.doi.org/10.1016/S0924-4247(02)00044-4

Hayashi, T., Kawashima, K., Sun, Z.Q., et al., 2005. Guided wave focusing mechanics in pipe. Journal of Pressure Vessel Technology, 127(3):317-321. http://dx.doi.org/10.1115/1.1990209

Hirao, M., Ogi, H., 2003. EMATs for Science and Industry: Noncontacting Ultrasonic Measurements. Kluwer Academic, Boston, USA. http://dx.doi.org/10.1007/978-1-4757-3743-1

Kim, Y.G., Moon, H.S., Park, K.J., et al., 2011. Generating and detecting torsional guided waves using magnetostrictive sensors of crossed coils. NDT \& E International, 44(2):145-151. http://dx.doi.org/10.1016/j.ndteint.2010.11.006

Kim, Y.Y., Park, C., Cho, S.H., et al., 2005. Torsional wave experiments with a new magnetostrictive transducer configuration. The Journal of the Acoustical Society of America, 117(6):3459-3468. http://dx.doi.org/10.1121/1.1904304

Kwun, H., Bartels, K.A., 1998. Magnetostrictive sensor technology and its applications. Ultrasonics, 36(1-5): 171-178. http://dx.doi.org/10.1016/S0041-624X(97)00043-7

Lee, J.S., Kim, Y.Y., Cho, S.H., 2009. Beam-focused shearhorizontal wave generation in a plate by a circular magnetostrictive patch transducer employing a planar solenoid array. Smart Materials \& Structures, 18(1): 015009. http://dx.doi.org/10.1088/0964-1726/18/1/015009

Li, J., Rose, J.L., 2001a. Excitation and propagation of nonaxisymmetric guided waves in a hollow cylinder. The Journal of the Acoustical Society of America, 109(2): 457-464. http://dx.doi.org/10.1121/1.1315290

Li, J., Rose, J.L., 2001b. Implementing guided wave mode control by use of a phased transducer array. IEEE Transactions on Ultrasonics, Ferroelectrics and Frequency Control, 48(3):761-768. http://dx.doi.org/10.1109/58.920708

Li, J., Rose, J.L., 2002. Angular-profile tuning of guided waves in hollow cylinders using a circumferential phased array. IEEE Transactions on Ultrasonics, Ferroelectrics and Frequency Control, 49(12):1720-1729. http://dx.doi.org/10.1109/TUFFC.2002.1159849

Luo, W., 2005. Ultrasonic Guided Waves and Wave Scattering in Viscoelastic Coated Hollow Cylinder. PhD Thesis, The Pennsylvania State University, State College, USA.

Marty, P.N., 2002. Modelling of Ultrasonic Guided Wave Field Generated by Piezoelectric Transducers. PhD Thesis, Imperial College, London, UK.

Marzani, A., 2008. Time-transient response for ultrasonic guided waves propagating in damped cylinders. International Journal of Solids and Structures, 45(2526):6347-6368. http://dx.doi.org/10.1016/j.ijsolstr.2008.07.028

Moreau, L., Velichko, A., Wilcox, P.D., 2012. Accurate finite element modelling of guided wave scattering from 
irregular defects. NDT \& E International, 45(1):46-54. http://dx.doi.org/10.1016/j.ndteint.2011.09.003

$\mathrm{Mu}$, J., 2008. Guided Wave Propagation and Focusing in Viscoelastic Multilayered Hollow Cylinders. PhD Thesis, The Pennsylvania State University, State College, USA.

$\mathrm{Mu}$, J., Rose, J.L., 2008. Guided wave propagation and mode differentiation in hollow cylinders with viscoelastic coatings. The Journal of the Acoustical Society of America, 124(2):866-874. http://dx.doi.org/10.1121/1.2940586

Philtron, J.H., Rose, J.L., 2014. Mode perturbation method for optimal guided wave mode and frequency selection. Ultrasonics, 54(7):1817-1824. http://dx.doi.org/10.1016/j.ultras.2014.02.005

Ribichini, R., 2011. Modelling of Electromagnetic Acoustic Transducers. PhD Thesis, Imperial College London, London, UK.

Ribichini, R., Cegla, F., Nagy, P.B., et al., 2010. Quantitative modeling of the transduction of electromagnetic acoustic transducers operating on ferromagnetic media. IEEE Transactions on Ultrasonics, Ferroelectrics and Frequency Control, 57(12):2808-2817. http://dx.doi.org/10.1109/TUFFC.2010.1754

Ribichini, R., Cegla, F., Nagy, P.B., et al., 2011. Study and comparison of different EMAT configurations for $\mathrm{SH}$ wave inspection. IEEE Transactions on Ultrasonics, Ferroelectrics and Frequency Control, 58(12):25712581. http://dx.doi.org/10.1109/TUFFC.2011.2120

Ribichini, R., Cegla, F., Nagy, P.B., et al., 2012. The impact of magnetostriction on the transduction of normal bias field EMATs. NDT \& E International, 51:8-15. http://dx.doi.org/10.1016/j.ndteint.2012.06.004

Rose, J.L., 2014. Ultrasonic Guided Waves in Solid Media. Cambridge University Press, Cambridge, UK. http://dx.doi.org/10.1017/CBO9781107273610

Rose, J.L., Pelts, S.P., Quarry, M.J., 1998. A comb transducer model for guided wave NDE. Ultrasonics, 36(1-5):163169. http://dx.doi.org/10.1016/S0041-624X(97)00042-5

Shin, H.J., Rose, J.L., 1999. Guided waves by axisymmetric and non-axisymmetric surface loading on hollow cylinders. Ultrasonics, 37(5):355-363. http://dx.doi.org/10.1016/S0041-624X(99)00010-4

Sun, Z.Q., Zhang, L., Rose, J.L., 2005. Flexural torsional guided wave mechanics and focusing in pipe. Journal of Pressure Vessel Technology, 127(4):471-478. http://dx.doi.org/10.1115/1.2065587

Turcu, F.O., 2008. Development and Experimentation of Magnetostrictive Sensors for Inspection and Monitoring of Piping Systems. PhD Thesis, Università Di Pisa, Pisa, Italy.

Wilcox, P.D., 2003. Omni-directional guided wave transducer arrays for the rapid inspection of large areas of plate structures. IEEE Transactions on Ultrasonics, Ferroelectrics and Frequency Control, 50(6):699-709. http://dx.doi.org/10.1109/TUFFC.2003.1209557
Zhang, L., 2005. Guided Wave Focusing Potential in Hollow Cylinders. PhD Thesis, The Pennsylvania State University, State College, USA.

Zhang, X.W., Tang, Z.F., Lü, F.Z., 2014. Numerical simulation and experimental investigation on ultrasonic guided waves in multilayered pipes based on SAFE. Journal of Mechanical Engineering, 50(8):10-16. http://dx.doi.org/10.3901/JME.2014.08.010

Zhu, W.H., 2001. A finite element analysis of the time-delay periodic ring arrays for guided wave generation and reception in hollow cylinders. IEEE Transactions on Ultrasonics, Ferroelectrics and Frequency Control, 48(5):1462-1470.

http://dx.doi.org/10.1109/58.949757

\section{中文概要}

题 目: 弹性空心圆柱体中对称与非对称导波的磁致伸 缩换能器激励

目 的: 超声导波是一种有潜力的无损检测方法, 磁致 伸缩换能器是一种重要的导波换能器。利用经 典的简正模态展开法分析弹性空心圆柱体在磁 致伸缩载荷下的激励响应, 包括磁致伸缩局部 载荷、轴向阵列载荷和圆周阵列载荷。空心圆 柱体在磁致伸缩载荷作用下的扰动分析将为磁 致伸缩换能器以及换能器阵的进一步发展提供 理论依据。

创新点: 1. 利用简正模态展开方法, 讨论弹性空心圆柱 体在磁致伸缩载荷作用下的扰动分析和激励响 应; 2. 通过数值仿真和试验研究, 验证空心圆 柱体在磁致伸缩局部载荷、轴向阵列载荷和圆 周阵列载荷作用下不同的激励响应, 得到有效 的结论。

方 法: 1. 通过理论推导, 构建典型磁致伸缩换能器数 学模型 (公式 (15) 和 (17)) ; 2. 通过理论 推导, 建立弹性空心圆柱体在磁致伸缩局部载 荷、轴向阵列载荷和圆周阵列载荷作用下的波 源分析模型（公式（25 26）、（30 31）和 （34 35））, 求解得到典型载荷形式下的激励 响应（图 6 8 和 11 13）；3. 通过仿真模拟和试 验研究, 对空心圆柱体在不同磁致伸缩载荷作 用下的激励响应进行验证（图 14 21）。

结 论: 1. 磁致伸缩局部载荷能够激励产生同一族的导 波模态, 但是不具有良好的频率选择性和相速 度选择性, 轴对称载荷仅能激励轴对称模态; 2. 磁致伸缩轴向阵列载荷具有良好的频率选择 性和相速度选择性, 中心频率和中心相速度取 决于阵列单元间距，对导波模态控制非常有 益; 3. 磁致伸缩圆周阵列载荷能够激励周向阶 次为阵列单元数整数倍的导波模态。

关键词: 导波; 空心圆柱体; 简正模态展开; 磁致伸缩 